(2) Open Access Full Text Article

ORIGINAL RESEARCH

\title{
Serum C-Reactive Protein in Patients with Deficit Schizophrenia and the Relationship with Cognitive Function
}

Li-Hong $\operatorname{Pan}^{1} * *$

Ming Qian ${ }^{1, *}$

Weihua $\mathrm{Qu}^{2}$

Qin Tang ${ }^{2}$

Yuzhong Yan ${ }^{3}$

'Department of Psychiatry, Shanghai Pudong Nanhui Mental Health Center, Shanghai, People's Republic of China;

${ }^{2}$ Department of Clinical Laboratory,

Shanghai Prison General Hospital,

Shanghai, People's Republic of China;

${ }^{3}$ Department of Research, Shanghai

University of Medicine and Health

Science Affiliated Zhoupu Hospital,

Shanghai, People's Republic of China

*These authors contributed equally to this work
This article was published in the following Dove Press journal:

Neuropsychiatric Disease and Treatment

Background: Serum levels of C-reactive protein (CRP) were measured in patients with deficit schizophrenia (DS) to confirm the association between CRP level and cognitive performance and to determine whether CRP was a new biological indicator with the potential clinical applications in DS patients.

Methods: Three independent samples [41 DS and 50 non-deficit schizophrenia (NDS) and 30 sex- and age-matched healthy controls (HCs)] were recruited in our study. Serum CRP levels were measured by immunofluorescence. The Positive and Negative Syndrome Scale (PANSS) and alternative forms of the Repeatable Battery for the Assessment of Neuropsychological Status (RBANS) were also assessed. And the relationships between serum CRP levels and both PANSS and RBANS scores were finally analyzed.

Results: The results of serum CRP level were showed significantly different among the three groups and increased from the HCs to NDS patients to DS patients. There were also significant differences in the cognitive subdomain analyses among the three groups. Serum CRP levels were found positively correlated with total and negative PANSS scores, and showed negatively correlated with overall cognitive test scores in the DS samples.

Conclusion: Serum C-reactive protein levels and their association with cognitive performance were different between deficit schizophrenia and non-deficit schizophrenia samples, and higher serum CRP level was associated with worse cognitive performance in the DS patients. The results indicated that CRP could be a potential biomarker, and DS could be a distinct subset of schizophrenia.

Keywords: schizophrenia, deficit schizophrenia, C-reactive protein, inflammation, cognitive impairment

\section{Introduction}

Schizophrenia is a severe mental disorder characterized by positive and negative psychotic symptoms, cognitive dysfunction and functional decline, with a lifetime prevalence close to $1 \% .^{1}$ The etiology of schizophrenia remains vague, but it points to a combination of both genetics and environmental events. Cognitive dysfunctions are core features of schizophrenia that detrimentally affect functional outcomes. ${ }^{2}$ There has been an increasing amount of evidence suggesting that underlying inflammatory mechanisms are relevant to cognitive functioning. ${ }^{3}$ Peripheral inflammation might be related to cognitive deficits in schizophrenia. ${ }^{4}$ The contribution of inflammatory mechanism in schizophrenia has attracted much attention in recent years. 
Carpenter et al have described deficit schizophrenia (DS) as a relatively homogeneous subset of patients with schizophrenia with a more severe illness characterized by long-lasting and prominent negative symptoms, a higher degree of cognitive impairment, a greater decrement in frontal and temporal brain volumes, and worse psychosocial functioning when compared to nondeficit schizophrenia (NDS). ${ }^{5,6}$ Mounting evidence indicates that DS patients could be a distinct schizophrenia subgroup, which helped to reduce the heterogeneity in the classification of patients with this disease.

Given the heterogeneity of the symptoms in patients with schizophrenia and current treatment limitations, biomarkers may play an important role in the diagnosis, subtype stratification, and the assessment of treatment response. C-reactive protein (CRP), a well- characterized and standardized marker of inflammation, is considered to be useful in identifying schizophrenia patients who may be more highly inflamed, which could contribute to treatment choice. $^{7-9}$

Reports on serum CRP levels in patients with schizophrenia showed inconsistently. ${ }^{10-12}$ And at present, studies on serum CRP levels in DS patients and its relationship with cognitive function are scarce by now. Therefore, the purpose of this study is to explore whether there was a discrepancy in serum CRP level between DS patients, NDS patients and the healthy control group (HCS). At the same time, the relationship between serum CRP level and cognitive function in patients with DS was also evaluated.

\section{Methods}

\section{Participants}

We recruited 91 clinically stable schizophrenia patients (41 DS and 50 NDS) and $30 \mathrm{HCs}$ coming from three independent samples. Schizophrenia patients were recruited from the psychiatric rehabilitation unit of Pudong New Area Nanhui Mental Health Center, Shanghai, China. The inclusion criteria were as follows: 1) diagnosis of schizophrenia was determined by the Structured Clinical Interview for the DSM-IV-TR (SCID); 2) the patients had a chronic course of disease for at least 7 years, aged between 20 and 70 years; and 3) received a stable dose of oral antipsychotics at least 12 months before recruitment. The Mini-International Neuropsychiatric Interview (MINI) was used to screen, ${ }^{13}$ who were excluded if they had a past or present diagnosis of a major neuropsychiatric illness. Other exclusion criteria were as follows: 1) other current DSM-IV-TR Axis I diagnoses; 2) alcohol or other non-tobacco substance abuse or dependence within the previous 3 months; 3) diagnosis of dementia, intellectual disability disorder, or a major neurological disorder; and 4) medical disability affecting the ability to complete study procedures. The study protocol was approved by the Institutional Ethical Committee for clinical research of Pudong New Area Nanhui Mental Health Center and all participants provided written informed consent prior to participation. This study was conducted in accordance with the Declaration of Helsinki.

\section{Detection of Serum CRP Levels}

For the analysis of CRP levels, blood samples were drawn from all patients in the fasting state between 08:00 and 10:00 $\mathrm{h}$ after a 12 -h overnight fast. Immediately after collecting blood samples, CRP serum levels were measured using the immunofluorescence method by the manufacturer (Roche).

\section{Cognitive Assessment}

Cognitive assessments were conducted using the Repeatable Battery for the Assessment of Neuropsychological Status (RBANS). RBANS has previously shown good reliability and validity in patients with psychosis. ${ }^{14}$ It takes only approximately $30 \mathrm{~min}$ to complete, making it practical and feasible to use in practice. The cognitive domains included language, visuospatial/constructional, immediate memory, delayed memory, and attention.

The original scores of neuropsychological variables were converted to $T$-scores using the best available criteria in the corresponding manuals. ${ }^{15}$ According to the average $T$-scores of five cognitive domains, the final summary score defines the overall $T$-score of cognitive function.

\section{Procedures}

Healthy controls and patients underwent a comprehensive evaluation by senior research psychiatrists and trained in the research procedure, including the use of the psychiatric scales, after the participants completed the informed consent and demographics form. The next appointment involved neuropsychological assessment, carried out by the same investigators. The same neuropsychological battery was performed on all subjects. There were standard instructions for the management of each tool.

\section{Statistical Analyses}

Descriptive statistics (mean, standard deviation, frequency) and independent sample $T$-tests were analyzed with SPSS 
Table I Demographic and Clinical Characteristics of DS, NDS and HC

\begin{tabular}{|c|c|c|c|c|c|}
\hline & DS $(N=41)$ & NDS $(N=50)$ & $\mathrm{HC}(\mathrm{N}=30)$ & $F / T / X^{2}$ & $p$ \\
\hline Age & $32.24 \pm 15.85$ & $33.76 \pm 14.26$ & $35.78 \pm 10.22$ & 0.56 & 0.58 \\
\hline $\operatorname{Sex}(M / F)$ & $24 / 17$ & $26 / 24$ & $16 / 14$ & 0.41 & 0.81 \\
\hline Education (years) & $5.12 \pm 2.88^{\# \#}$ & $5.51 \pm 3.01^{\S}$ & $7.24 \pm 3.76$ & 4.25 & $<0.05$ \\
\hline Smoking ratio (\%) & 41.46 & 40.00 & 43.33 & 1.38 & 0.50 \\
\hline BMI $\left(\mathrm{kg} / \mathrm{m}^{2}\right)$ & $22.82 \pm 6.12$ & $23.24 \pm 7.23$ & $21.90 \pm 7.15$ & 0.36 & 0.70 \\
\hline CRP & $14.32 \pm 12.26$ *\#\# & $9.87 \pm 6.95^{\S \S}$ & $4.39 \pm 2.16$ & 11.84 & $<0.001$ \\
\hline Age of onset (years) & $21.12 \pm 5.88$ & $23.28 \pm 8.79$ & & 1.35 & 0.18 \\
\hline Duration of illness (years) & $20.19 \pm 6.41$ & $18.92 \pm 7.16$ & & 0.88 & 0.38 \\
\hline PANSS total & $70.98 \pm 11.09$ & $57.08 \pm 11.88$ & & 5.72 & $<0.001$ \\
\hline PANSS positive & $16.56 \pm 4.48$ & $15.16 \pm 5.62$ & & 1.29 & 0.19 \\
\hline PANSS negative & $21.98 \pm 7.46$ & $12.50 \pm 3.65$ & & 7.91 & $<0.001$ \\
\hline PANSS general & $32.44 \pm 7.88$ & $29.42 \pm 9.62$ & & 1.61 & 0.11 \\
\hline RBANS T-score & $30.61 \pm 8.74$ & $39.66 \pm 9.27$ & & 4.75 & $<0.001$ \\
\hline \multicolumn{6}{|l|}{ Antipsychotic treatment } \\
\hline Olanzapine $(\mathrm{N})$ & 25 & 28 & & 0.76 & 0.68 \\
\hline Risperidone $(\mathrm{N})$ & 15 & 19 & & & \\
\hline Aripiprazole (N) & I & 3 & & & \\
\hline Chlorpromazine equivalents (mg/day) & $523.06 \pm 198.76$ & $497.34 \pm 229.32$ & & 0.56 & 0.57 \\
\hline
\end{tabular}

Notes: ${ }^{\#} \mathrm{p}<0.001$ DS vs HC; ${ }_{\mathrm{p}}^{\S}<0.05$ NDS vs HC; ${ }^{\S \S} \mathrm{p}<0.001$ NDS vs HC; ${ }^{*} \mathrm{p}<0.05$ DS vs NDS.

version 24.0. The continuous variables of demographic, clinical and cognitive raw data are presented as the mean with standard deviation and analyzed. Pearson correlation coefficient was used to evaluate the relationship between parameter variables, and Spearman correlation analysis was used to evaluate categorical variables. Bonferroni correction was performed on each test to adjust multiple tests. The chisquare test was used to analyze statistical relationships of categorical variables. For all analyses, results were considered statistically significant for $\mathrm{p}<0.05$.

\section{Results}

\section{Demographic Data}

Table 1 shows the basic demographic data in controls and both groups of schizophrenia. There were no significant differences in age, sex, smoking and/or body mass index (BMI) among the three study groups. Post hoc comparisons revealed less education for DS and NDS patients than in the HCs, while the two patient subgroups did not significantly differ. The results also showed that there were no significant differences in age of onset, course of disease and dosage of antipsychotics (chlorpromazine equivalent) between DS and NDS patients. There was no significant difference in the types of antipsychotic treatment between the DS and NDS groups. The DS patients showed more severe total psychopathological symptoms and negative symptoms than the NDS patients, but not in either positive or general syndrome regarding PANSS scores (Figure 1A).

\section{Cognitive Performance in Patients with Schizophrenia and HCs}

Global cognition was significantly impaired in the patients with DS in comparison with NDS using the RBANS (Table 1, Figure 1B). The same as for the RBANS, performance on the five cognitive subdomains tests is summarized in Table 2. The cognition performance of the DS group was significantly worse than that of the NDS group in all five cognitive tests. 

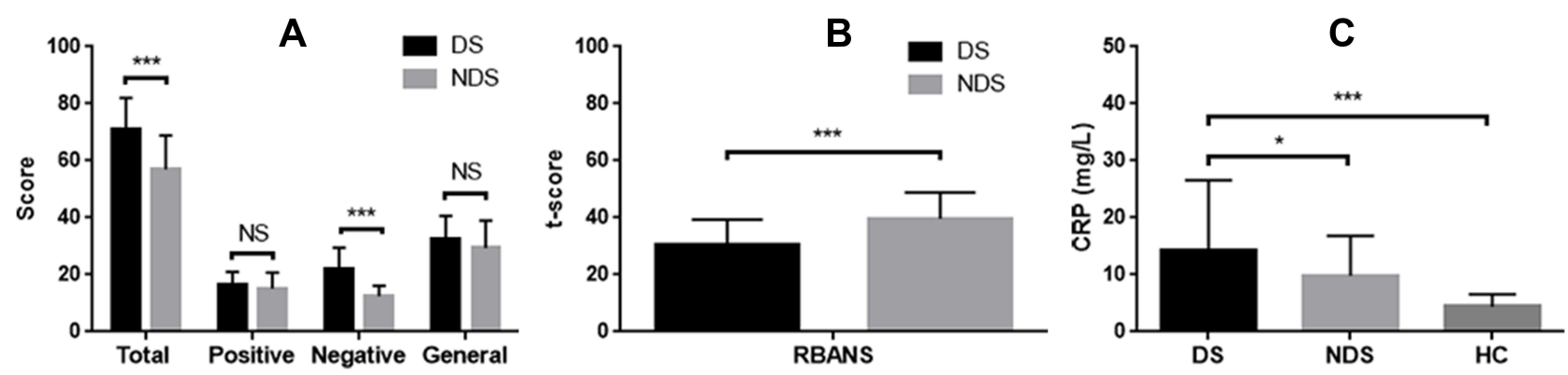

Figure I (A) The DS patients showed more severe psychopathological total symptom $(F=5.72$, *** $p<0.00 \mathrm{I})$ and negative symptoms $(F=7.9 \mathrm{I}$, $* * * p<0.00 \mathrm{I})$ than NDS, but not in either positive $\left(F=1.29,{ }_{p}{ }_{p}=0.19\right)$ or general syndrome $\left(F=1.61,{ }^{N S}=0.11\right)$ regarding PANSS scores. (B) RBANS showed a lower T-score in patients with DS in comparison with NDS $(T=4.75$, *** $p<0.00 \mathrm{I})$. (C) Serum CRP levels were significantly different in the three study groups $(F=1 \mathrm{I} .84$, $* * * p<0.00 \mathrm{I})$, and DS patients showed a higher level than NDS patients $\left(T=2.18,{ }^{*} p=0.03\right)$.

\section{Serum CRP in Patients with}

\section{Schizophrenia and $\mathrm{HCs}$}

The serum CRP levels were significantly different among the three groups (Table 1, Figure 1C). DS patients showed significantly higher levels of serum CRP than NDS patients.

\section{The Relationships Between Serum CRP Levels and PANSS Scores or Cognitive Performance}

Pearson correlation tests of the relationships were performed between serum CRP levels and PANSS score (Figure 2), or overall cognitive performance (Figure 3), respectively. A positive relationship was found between serum CRP levels and PANSS total and negative scores among the DS patients (Figure 2). And there was a statistically significant negative relationship between serum CRP levels and overall cognitive performance (Figure 3). Interestingly, the association relationships were not found among the NDS patients in this study (Figures 2 and 3).

\section{Discussion}

The first major result of this study was revealed that serum CRP levels are negatively correlated with overall cognitive performance was shown only among DS patients. This relationship association was especially useful for the subset diagnosis of schizophrenia. In addition, it could be considered a biomarker capable of discriminating DS from NDS patients. Furthermore, analysis of the PANSS subscale scores confirmed that significant differences were found in total and negative scores between the DS and NDS patients, while there were no differences in the positive and general scores. Consistent with previous studies ${ }^{16}$ this study provided evidence for the hypothesis that DS is a unique group or pathological entity. The separation of deficits from NDS can provide a more homogeneous phenotype, and thus may enable the identification of pathophysiological mechanisms underpinning this complex phenotype.

Although the pathophysiology of schizophrenia is still not completely understood, there is accumulating evidence that dysregulation in components of the immune system are fundamentally linked to the disease. ${ }^{17,18}$ It has been proposed that chronic inflammation might damage the microvascular system of the brain, hamper cerebral blood flow, and trigger the expression of genes that cause chronic, low-grade inflammation. ${ }^{19,20}$ Researchers have pointed out that increasing CRP levels were associated with increasing risk for psychological distress. ${ }^{21}$ Thus, studies have been conducted to decipher the potentiality of CRP as a marker for inflammation in schizophrenia. ${ }^{19}$

Table 2 Comparisons of the Cognitive Subdomains Among DS, NDS and HC Groups

\begin{tabular}{|l|l|l|l|l|l|}
\hline & DS & NDS & HC & F \\
\hline Verbal abilities & $34.10 \pm 8.85^{\# \#_{* *}}$ & $40.10 \pm 8.86^{\S \S}$ & $72.22 \pm 23.17$ & $73.7 I$ & P \\
Visuospatial abilities & $38.55 \pm 11.23^{\# \# * *}$ & $47.10 \pm 12.87^{\S \S}$ & $75.10 \pm 12.88$ & 80.55 & $<0.001$ \\
Learning & $23.18 \pm 5.17^{\# \#_{* *}}$ & $35.80 \pm 8.18^{\S \S}$ & $61.18 \pm 10.29$ & 201.7 & $<0.001$ \\
Memory & $27.12 \pm 9.34^{\# \#_{* *}}$ & $38.24 \pm 9.89^{\S}$ & $63.21 \pm 11.90$ & 113.6 & $<0.001$ \\
Attention & $21.74 \pm 6.12^{\# \#_{* *}}$ & $29.70 \pm 7.90^{\S \S}$ & $53.64 \pm 9.91$ & $<0.001$ \\
\hline
\end{tabular}

Notes: ${ }^{\#} \mathrm{p}<0.001$ DS vs HC; ${ }^{\S \S} \mathrm{p}<0.001$ NDS vs HC; ${ }^{* *} \mathrm{p}<0.00$ I DS vs NDS. 

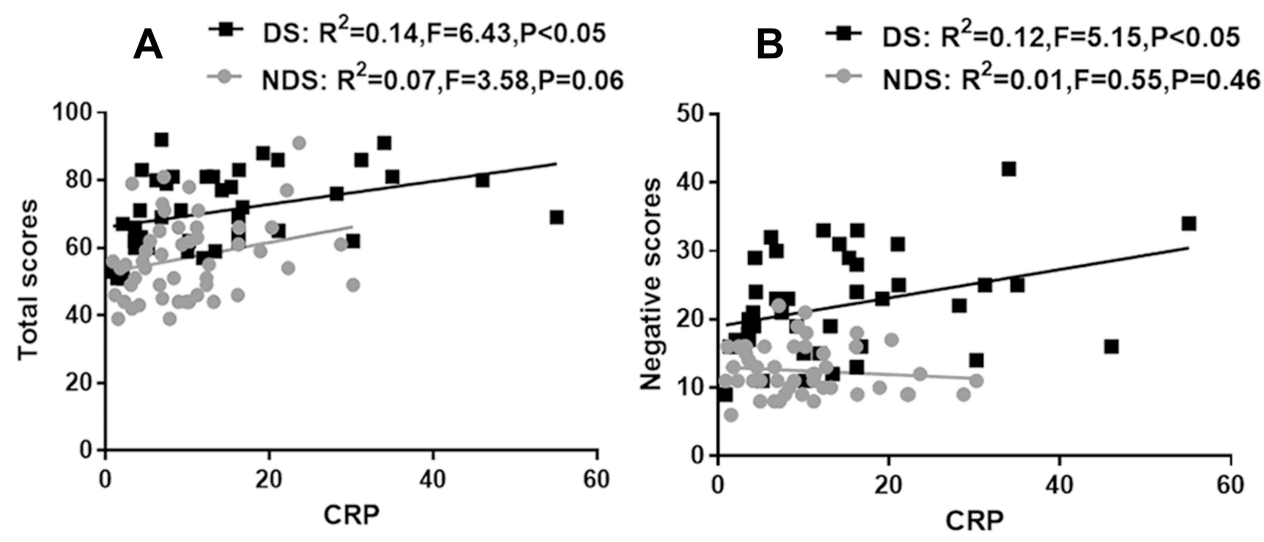

Figure 2 Association between CRP level and PANSS score. (A) Scatter plot of CRP level versus Total scores $\left((\mathbf{A})\right.$ DS patients: $R^{2}=0.14, F=6.43, \mathrm{P}<0.05$; NDS patients: $R^{2}$ $=0.07, F=3.58, \mathrm{P}=0.06$ ), and negative scores $\left((\mathrm{B}) \mathrm{DS}\right.$ patients: $R^{2}=-0.12, F=5.15, \mathrm{P}<0.05 ;$ NDS patients: $\left.R^{2}=0.0 \mathrm{I}, F=0.55, \mathrm{P}=0.46\right)$.

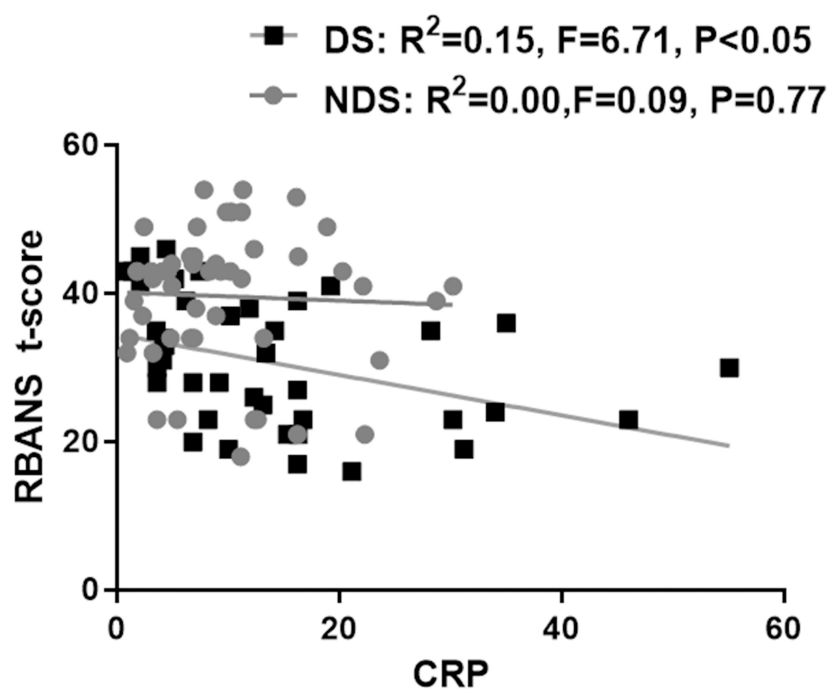

Figure 3 Association relationship between CRP level and BRANS T-score. Scatter plot of CRP level versus BRANS T-score among DS $\left(R^{2}=0.15, F=6.71, P<0.05\right)$ and NDS $\left(R^{2}=0.00, F=0.09, P=0.77\right)$.

The results of these studies have mostly been conclusive and show increased levels of CRP in patients with these disorders. However, fewer studies have compared CRP levels in these DS and NDS diagnostic groups. In this study, serum CRP levels were significantly higher in the DS patients than in the NDS patients or the controls. Based on these findings, we also suggested considering the possibility of monitoring the serum CRP levels to roughly evaluate patients with schizophrenia.

Evidence has indicated that DS patients may present more severe features as reflected by more negative symptoms and more severe neurocognitive impairments compared to NDS patients. ${ }^{16}$ Researchers have also found that patients with DS might present higher levels of a subthreshold inflammatory state in terms of elevated CRP levels that may be associated with their negative symptoms. ${ }^{22}$ As expected, our results indicated that the DS patients showed more severe psychopathological features than the NDS patients. Significant differences in total and negative PANSS scores and overall cognitive performance were found between the DS and NDS subsets. Furthermore, our results reflected associations between differences in CRP levels and the mental disorders. The results were consistent with previous studies that suggested that serum CRP levels could be a biomarker to differentiate "deficit" and "non-deficit" subtypes.

In this study, the associations between serum CRP levels and PANSS items and cognitive performance were evaluated. Through correlation analyses, it was shown that serum CRP levels were positively correlated with PANSS total and negative scores in the DS patients. It was also shown in our study that an inverse association between CRP serum levels and cognitive performance was presented. The results suggested that serum CRP levels could reflect, to a certain extent, the severe clinical symptoms of DS patients. Reports have indicated that the serum levels of inflammatory markers decrease with improvements in negative symptoms and cognitive impairment function. ${ }^{23}$ It is speculated that CRP plays an important role not only in the pathogenesis of inflammation but also in the pathogenesis of mental disorders. Fathian et al discovered that initial changes in the serum levels of CRP during the acute phase of psychosis could predict cognitive function in the later phases of the disease. ${ }^{24}$ Although the underlying mechanisms need to be further elucidated, CRP 
could play a role in the development of this psychiatric disorder.

In summary, this study showed that serum CRP levels were significantly higher in DS patients than in NDS patients and were associated with the condition and cognitive function of DS patients. However, our findings should be interpreted with caution, and further investigation in larger samples to determine whether serum CRP levels cloud be used as a potential biological indicator in disease diagnosis and differentiation, thereby guiding clinical therapy and prognosis in DS patients is warranted.

\section{Author Contributions}

All authors made substantial contributions to conception and design, acquisition of data, or analysis and interpretation of data; took part in drafting the article or revising it critically for important intellectual content; agreed to submit to the current journal; gave final approval of the version to be published; and agree to be accountable for all aspects of the work.

\section{Funding}

This study was supported by grants from Shanghai University of Medicine and Health Science (SFP-18-2117-002), by grants from Pudong Health Bureau of Shanghai (PWRd2019-03), the Outstanding Clinical Discipline Project of Shanghai Pudong (PWYgy201803), the Research Grant for Science and Technology Commission of Pudong New Area of Shanghai (PKJ2019Y61), and Shanghai Key Medical Speciality Construction Plan (ZK2019B25).

\section{Disclosure}

The authors report no conflicts of interest for this work and have no conflict of interest with any commercial or other association in connection with the submitted article.

\section{References}

1. Tandon R, Nasrallah HA, Keshavan MS. Schizophrenia, "just the facts" 4. Clinical features and conceptualization. Schizophr Res. 2009;110(1-3):1-23. doi:10.1016/j.schres.2009.03.005

2. Kahn RS, Keefe RS. Schizophrenia is a cognitive illness: time for a change in focus. JAMA Psychiatry. 2013;70(10):1107-1112. doi:10.1001/jamapsychiatry.2013.155

3. Kroken RA, Loberg EM, Dronen T, et al. A critical review of pro-cognitive drug targets in psychosis: convergence on myelination and inflammation. Front Psychiatry. 2014;5:11. doi:10.3389/ fpsyt.2014.00011
4. Misiak B, Stańczykiewicz B, Kotowicz K, Rybakowski JK, Samochowiec J, Frydecka D. Cytokines and C-reactive protein alterations with respect to cognitive impairment in schizophrenia and bipolar disorder: a systematic review. Schizophr Res. 2018;192:16-29. doi:10.1016/j.schres.2017.04.015

5. Kirkpatrick B, Mucci A, Galderisi S. Primary, enduring negative symptoms: an update on research. Schizophr Bull. 2017;43 (4):730-736. doi:10.1093/schbul/sbx064

6. Carpenter WT, Heinrichs DW, Wagman AM. Deficit and nondeficit forms of schizophrenia: the concept. Am J Psychiatry. 1988;145 (5):578-583.

7. Nimgaonkar VL, Dickerson F, Pouget JG, et al. Joint evaluation of serum C-reactive protein levels and polygenic risk scores as risk factors for schizophrenia. Psychiatry Res. 2018;261:148-153.

8. Goldsmith DR, Crooks CL, Walker EF, Cotes RO. An update on promising biomarkers in schizophrenia. Focus (American Psychiatric Publishing). 2018;16(2):153-163.

9. Carrizo E, Fernandez V, Quintero J, et al. Coagulation and inflammation markers during atypical or typical antipsychotic treatment in schizophrenia patients and drug-free first-degree relatives. Schizophr Res. 2008;103(1-3):83-93. doi:10.1016/j.schres.2008.03.004

10. Orsolini L, Sarchione F, Vellante F, et al. Protein-C reactive as biomarker predictor of schizophrenia phases of illness? A systematic review. Curr Neuropharmacol. 2018;16(5):583-606. doi:10.2174/1570159X16666180119144538

11. Bulzacka E, Boyer L, Schurhoff F, et al. Chronic peripheral inflammation is associated with cognitive impairment in schizophrenia: results from the multicentric FACE-SZ dataset. Schizophr Bull. 2016;42(5):1290-1302. doi:10.1093/schbul/sbw029

12. Joseph J, Depp C, Martin AS, et al. Associations of high sensitivity C-reactive protein levels in schizophrenia and comparison groups. Schizophr Res. 2015;168(1-2):456-460. doi:10.1016/j. schres.2015.08.019

13. Sheehan DV, Lecrubier Y, Sheehan KH, et al. The mini-international neuropsychiatric interview (M.I.N.I.): the development and validation of a structured diagnostic psychiatric interview for DSM-IV and ICD-10. J Clin Psychiatry. 1998;59(Suppl 20):22-33;quiz34-57.

14. Wilk CM, Gold JM, Bartko JJ, et al. Test-retest stability of the repeatable battery for the assessment of neuropsychological status in schizophrenia. Am $J$ Psychiatry. 2002;159(5):838-844. doi:10.1176/appi.ajp.159.5.838

15. Randolph C, Tierney MC, Mohr E, Chase TN. The rEpeatable Battery for the Assessment of Neuropsychological Status (RBANS): preliminary clinical validity. J Clin Exp Neuropsychol. 1998;20 (3):310-319. doi:10.1076/jcen.20.3.310.823

16. Kanchanatawan B, Sriswasdi S, Thika S, et al. Deficit schizophrenia is a discrete diagnostic category defined by neuro-immune and neurocognitive features: results of supervised machine learning. Metab Brain Dis. 2018;33(4):1053-1067. doi:10.1007/s11011-018-0208-4

17. Brown AS, Derkits EJ. Prenatal infection and schizophrenia: a review of epidemiologic and translational studies. Am J Psychiatry. 2010;167 (3):261-280. doi:10.1176/appi.ajp.2009.09030361

18. Miller BJ, Buckley P, Seabolt W, Mellor A, Kirkpatrick B. Metaanalysis of cytokine alterations in schizophrenia: clinical status and antipsychotic effects. Biol Psychiatry. 2011;70(7):663-671. doi:10.1016/j.biopsych.2011.04.013

19. Singh B, Chaudhuri TK. Role of C-reactive protein in schizophrenia: an overview. Psychiatry Res. 2014;216(2):277-285. doi:10.1016/j. psychres.2014.02.004

20. Hanson DR, Gottesman II. Theories of schizophrenia: a genetic-inflammatory-vascular synthesis. BMC Med Genet. 2005;6:7.

21. Wysokinski A, Margulska A, Strzelecki D, Kloszewska I. Levels of C-reactive protein (CRP) in patients with schizophrenia, unipolar depression and bipolar disorder. Nord J Psychiatry. 2015;69 (5):346-353. doi:10.3109/08039488.2014.984755 
22. Garcia-Rizo C, Fernandez-Egea E, Oliveira C, Justicia A, Bernardo M, Kirkpatrick B. Inflammatory markers in antipsychotic-naive patients with nonaffective psychosis and deficit vs. nondeficit features. Psychiatry Res. 2012;198(2):212-215. doi:10.1016/j.psychres.2011.08.014

23. Chen X, Xiong Z, Li Z, et al. Minocycline as adjunct therapy for a male patient with deficit schizophrenia. Neuropsychiatr Dis Treat. 2018;14:2697-2701. doi:10.2147/NDT.S179658
24. Fathian F, Loberg EM, Gjestad R, et al. Associations between C-reactive protein levels and cognition during the first 6 months after acute psychosis. Acta Neuropsychiatr. 2019;31(1):36-45. doi:10.1017/neu.2018.25

\section{Publish your work in this journal}

Neuropsychiatric Disease and Treatment is an international, peerreviewed journal of clinical therapeutics and pharmacology focusing on concise rapid reporting of clinical or pre-clinical studies on a range of neuropsychiatric and neurological disorders. This journal is indexed on PubMed Central, the 'PsycINFO' database and CAS, and is the official journal of The International Neuropsychiatric Association (INA). The manuscript management system is completely online and includes a very quick and fair peer-review system, which is all easy to use. Visit http://www.dovepress.com/testimonials.php to read real quotes from published authors.

Submit your manuscript here: https://www.dovepress.com/neuropsychiatric-disease-and-treatment-journal 